\section{Clinical management} of polycystic kidney

\section{disease}

Nick Hateboer MD MRCP, Consultant Nephrologist, Dorset County Hospital, Dorchester, Dorset

Clin Med 2003;3:509-12

Autosomal dominant polycystic kidney disease $(\mathrm{ADPKD})$ is a systemic disease with renal and various extrarenal manifestations. It is one of the most common hereditary disorders, affecting approximately one in 1,000 individuals and currently accounts for $6.5 \%$ of end-stage renal disease (ESRD) in England and Wales.

\section{Genetics}

ADPKD is caused by mutations in two genes: ${ }^{1,2}$

- PKD1 on chromosome 16p13.3, and

- PKD2, on chromosome 4q21-23.
The proteins encoded by PKD1 and PKD2 are polycystin-1 and polycystin-2 (Fig 1)..$^{3}$ Polycystin-1 is probably involved in protein-protein or proteincarbohydrate interactions. Polycystin-2 is a nonselective cation channel that can conduct calcium ions. Mutations in either PKD1 or PKD2 produce identical clinical manifestations and polycystin-1 and polycystin-2 may be part of a common pathway. Although PKD1 and PKD2 are clinically indistinguishable, PKD2 mutations are associated with fewer complications and a longer renal survival (Fig 2). ${ }^{4}$

\section{Diagnosis}

ADPKD is usually diagnosed with ultrasound. The diagnostic criteria for PKD1 are well established ${ }^{5}$ (Table 1 ). The criteria are less clear for $P K D 2$, but the PKD1 ultrasound criteria can be used for individuals older than 30 . For individuals younger than 30 , there is a need to be aware of occasional false negative scans. Linkage analysis can confirm or refute the diagnosis in cases of doubt, but not all families are suitable for this type of analysis, which requires the collection of DNA samples from several other

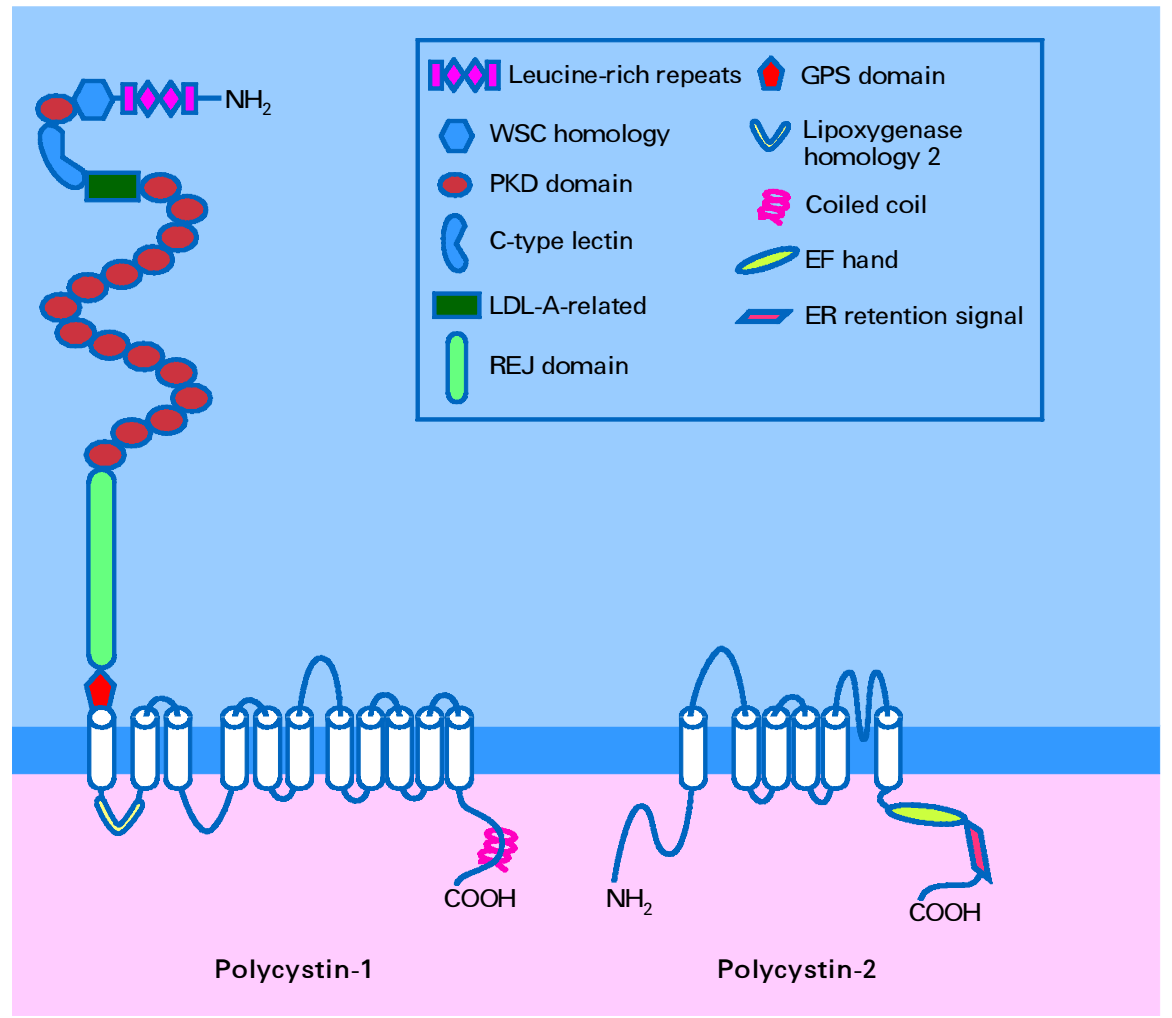

living affected and unaffected family members. Screening for mutations in the large PKD1 and PKD2 genes by DNA sequencing is not yet readily available in the UK.

\title{
Screening
}

Screening of asymptomatic family members and children poses an ethical dilemma. Screening might detect a treatable complication, such as hypertension, but at present there is no cure for ADPKD and confirmation of the diagnosis can lead to a degree of stigmatisation in an otherwise healthy individual. There may be repercussions for employment and life insurance. Currently, the general consensus is to avoid screening of asymptomatic children and to wait until they reach the age of consent. Ideally, even ultrasound scanning should be preceded by genetic counselling.

\section{Clinical features}

\section{Hypertension}

Hypertension is the presenting clinical finding in $13-20 \%$ of patients and frequently develops before renal function becomes impaired. Observational studies show that faster decline in renal function is associated with hypertension, but there is no conclusive trial evidence that treatment of hypertension reduces the rate of decline. ${ }^{6}$ Angiotensin-converting enzyme inhibitors (ACEI) and angiotensin II receptor blockers are known to reduce the rate of loss of kidney function in proteinuric patients with both diabetic and nondiabetic renal disease. In ADPKD (a nonproteinuric renal disease), there is no evidence that these drugs are better at protecting against decline of renal function. However, they may still have an

Fig 1. Structures of polycystin-1 and polycystin-2 (thick gray line = membrane bilayer; tan background = cytosol; blue background = extracellular space or endoplasmatic reticulum (ER) lumen; light grey cylinders $=$ putative transmembrane segments) (GPS $=$ G protein-coupled receptor proteolytic site; LDL-A = low-density lipoprotein apheresis; PKD = polycystic kidney disease; REJ = receptor egg jelly) ${ }^{3}$ 


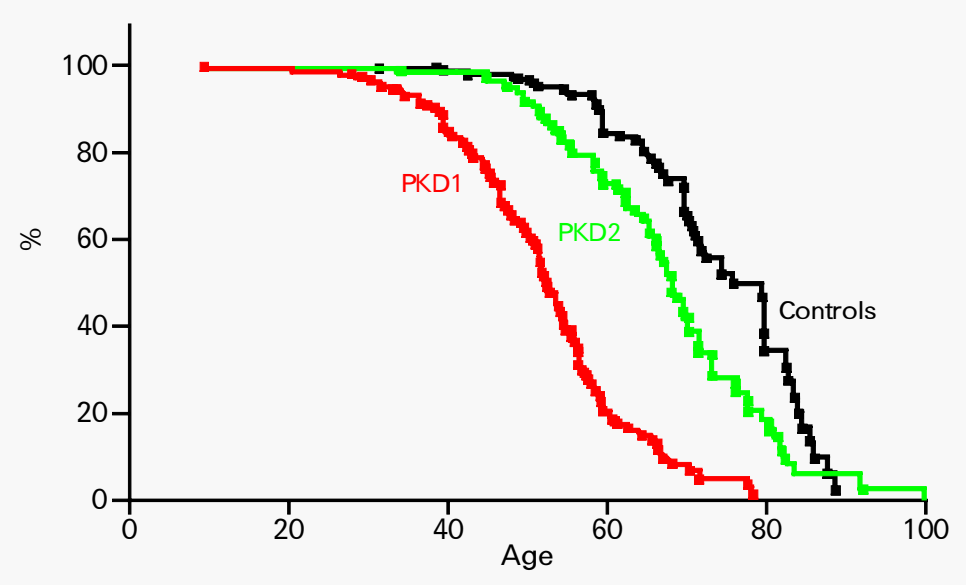

\section{Median age}

PKD1 $53.0(51.2-54.8)$

PKD2 $69.1(66.9-71.3)$

Controls $78.0(73.8-82.2)$ advantage over some other antihypertensive classes in reducing the development of left ventricular hypertrophy (LVH).

\section{Left ventricular hypertrophy}

The leading cause of death in ADPKD is cardiovascular disease. ${ }^{7}$ LVH is well established as a risk factor for cardiovascular morbidity and mortality; its incidence is increased in ADPKD, most probably a result of early untreated hypertension. In a large, seven-year, prospective randomised study ${ }^{8}$ comparing the impact of rigorous $(<120 / 80 \mathrm{mmHg})$ versus standard (135-140/85-90 mmHg) blood pressure control on LVH and renal function, the former was more effective in decreasing LVH. However, as in the Modification of Diet in Renal Disease study, ${ }^{6}$ there was no difference in renal function decline.

\section{Haematuria}

Frank haematuria is common in ADPKD. It often occurs spontaneously, but is occasionally associated with urinary tract infection, strenuous activity and, rarely, with kidney stones. The incidence is related to kidney size, the pres- ence of hypertension and the degree of renal impairment. Gross haematuria usually ceases within several days and rarely requires specific medical or surgical intervention. Clinicians should remain wary of missing alternative causes, particularly bladder cancer.

\section{Pain}

Flank or abdominal pain is experienced by at least $50-60 \%$ of patients with ADPKD at some time during their life. Chronic pain is most probably related to cyst distension, whereas acute pain is more suggestive of intracystic haemorrhage, infection or urinary tract obstruction. Most patients with chronic pain are helped by simple analgesics, but sometimes surgical decompression or percutaneous intracystic injection of a sclerosing agent may be considered.

\section{Renal calculi}

The estimated prevalence of renal calculi in ADPKD is $11-34 \%$, but they are often asymptomatic. Uric acid can be found in $57 \%$ of stones and calcium oxalate in $47 \%$. Options for treatment and prophy-

\section{Table 1. Ultrasound criteria.}

\section{Age (years) Criteria}

$\begin{array}{ll}<30 & \text { at least } 2 \text { renal cysts (unilateral or bilateral) } \\ 30-59 & \text { at least } 2 \text { cysts in each kidney } \\ \geq 60 & \text { at least } 4 \text { cysts in each kidney }\end{array}$




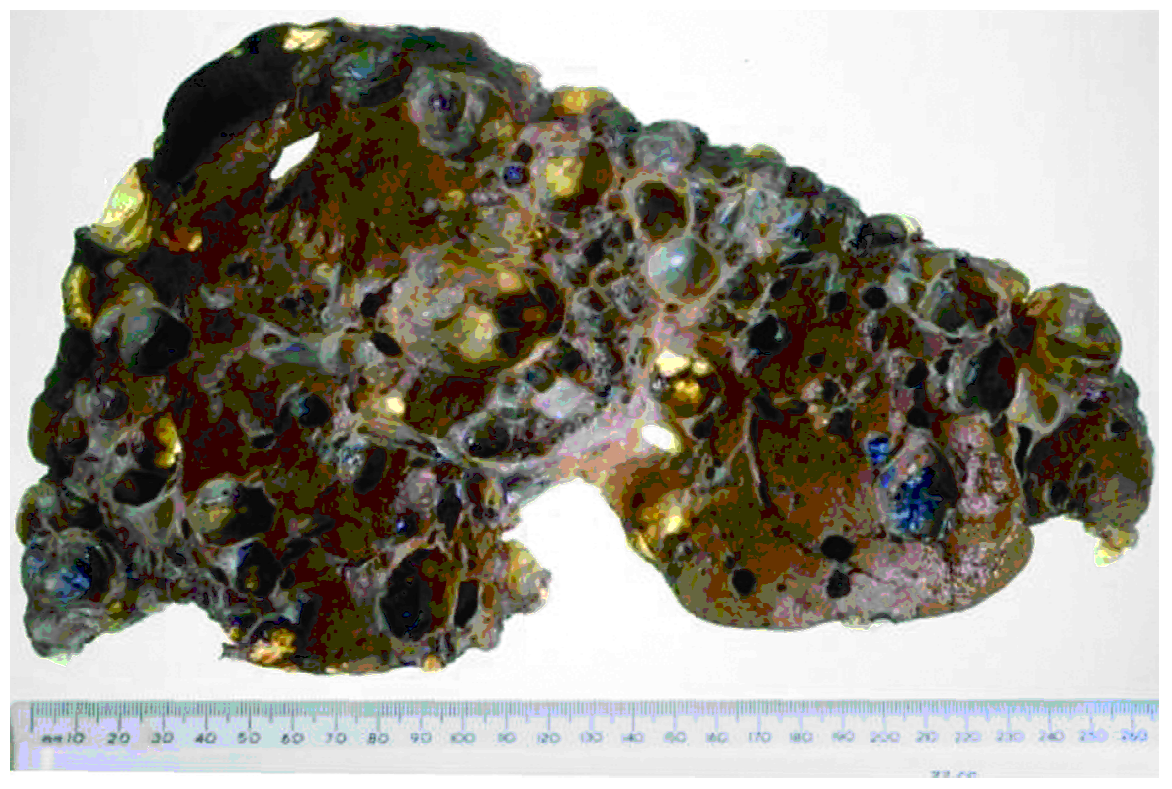

Fig 3. Massive cystic hepatomegaly in a patient requiring combined kidney and liver transplantation (from Ref 9 with permission).

\section{Extrarenal manifestations}

\section{Cysts}

Cysts can also be identified in the liver, spleen, pancreas and brain (arachnoid cysts) and occasionally in the oesophagus, ovaries, uterus and seminal vesicles. Liver cysts are common, with a higher prevalence in women. Pregnancy is a risk factor for massive hepatic cystic involvement (Fig 3), thought to be related to oestrogens. Some authors have therefore suggested that hormone replacement therapy should be avoided in women with liver cysts. Liver cysts are usually asymptomatic, but occasionally give rise to abdominal pain, symptoms due to compression of the gastrointestinal tract, obstructive jaundice, portal hypertension or compression of the intrahepatic vena cava.

\section{Intracranial aneurysms}

The association between ADPKD and intracranial aneurysms (ICAs) is well established. The prevalence of asymptomatic ICA in the ADPKD population is
Fig 4. Algorithm for screening for intracranial aneurysm (CT = computed tomography; ICA = intracranial aneurysm; $\mathrm{MR}=$ magnetic resonance) (from Yves Pirson, with permission).

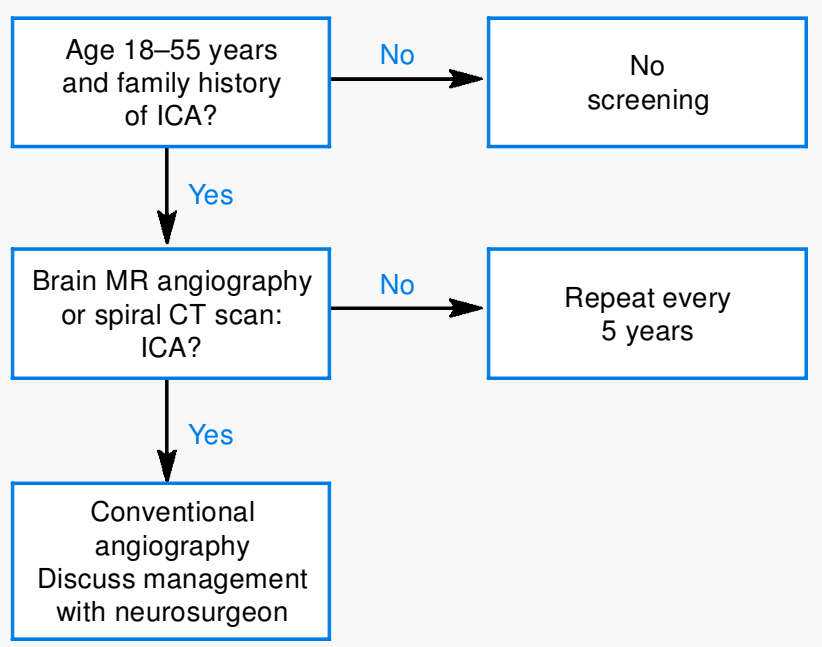

about $8 \%, 4-5$ times higher than in the general population; ${ }^{10}$ it may be as high as $25 \%$ among those with a family history of ICA. Rupture of ICA accounts for approximately $6 \%$ of all deaths in $\mathrm{ADPKD}^{7}$ and also occurs at a younger age than in the general population. Screening for ICAs with magnetic resonance angiography (MRA) might be beneficial for certain categories of patients, but it remains unclear precisely who should be screened with MRA. However, there is consensus that those with a family history of brain haemorrhage should be offered screening (Fig 4).

\section{Cardiac valvular/cardiovascular pathology}

ADPKD is associated with various cardiac valve abnormalities, including mitral incompetence, mitral valve prolapse, tricuspid incompetence, tricuspid valve prolapse and aortic incompetence. Other reported cardiovascular abnormalities in ADPKD include coronary artery aneurysms, atrial-septal aneurysms, diastolic dysfunction, dissection of the vertebral artery, aortic dissection and LVH. The prevalence of abdominal aortic aneurysms is probably not increased in ADPKD.

\section{Hernia and other connective tissue problems}

Inguinal and umbilical hernias are commonly found in ADPKD. Taken together with the valvular and cardiovascular complications, it is clear that ADPKD should be considered a connective tissue disorder with similarities to conditions like Marfan and Ehlers-Danlos syndromes.

\section{Diverticular disease}

There have been numerous case reports of complications of diverticular disease in patients with ADPKD and it has been suggested that the incidence is increased in ADPKD. However, there was a similar prevalence of diverticular disease in the patients and the healthy controls in the only controlled study in which 55 patients with ADPKD underwent a barium enema. ${ }^{11}$ 


\section{Renal cell carcinoma}

The prevalence of renal cell carcinoma (RCC) is not increased in ADPKD. ${ }^{12}$ However, RCC is more often concurrently bilateral, multicentric and sarcomatoid in type than in the general population. ${ }^{13}$ In addition, the diagnosis is often difficult and delayed. A recent large international study confirmed that there was no undue excess risk of kidney cancer in patients with ESRD due to ADPKD. ${ }^{11}$

\section{Key Points}

Autosomal dominant polycystic kidney
disease (ADPKD) is a systemic
disease with various extrarenal
manifestations

Subarachnoid haemorrhage from intracranial aneurysms is the most serious extrarenal complication; screening with magnetic resonance angiography should be offered to ADPKD patients with a family history of brain haemorrhage

Mutations in two different genes, PKD1 on chromosome 16 and PKD2 on chromosome 4 cause ADPKD. Clinically, the two conditions are indistinguishable, but overall PKD1 is more severe

The diagnosis of ADPKD still relies on ultrasound, with clearly defined criteria according to the number of renal cysts. False negative scans occasionally occur under the age of 30

\section{Hypertension and left ventricular hypertrophy are common early complications; cardiovascular disease is the leading cause of death in ADPKD}

Kidney failure generally occurs around age 55 . There is no specific treatment which is known to slow down the decline in renal function

In contrast to other renal conditions, angiotensin-converting enzyme inhibitors are not superior to other drugs in slowing progression to renal failure in ADPKD

KEY WORDS: autosound dominant, polycystic kidney

\section{Treatment}

Many dietary and pharmacological intervention strategies have been studied in humans and animals with PKD in an effort to slow the rate of renal progression. To date, no strategy has shown a consistent benefit in preserving renal function in humans with PKD. ${ }^{14}$ More encouraging are data from a recent epidemiological study which compared survival to ESRD in patient cohorts from 1985-1992 and from 1992-2001. The latter cohort had lower blood pressures, increased use of ACEIs and slower progression of renal decline. ${ }^{15}$

\section{Future prospects}

New insights into the molecular genetics of ADPKD and improved understanding of the pathophysiological processes necessary for cyst development may help in the development of novel pharmacological and other interventions. An exciting discovery has been that both polycystins, 1 and 2, are expressed in renal cilia. ${ }^{16}$ Cilia are long, thin tubular structures on the surface of renal tubular cells. Their function in human kidneys is unknown but they may have a chemosensory or mechanosensory function. This discovery has opened another avenue for research. When the function of these cilia becomes clearer, they may provide further therapeutic opportunities.

\section{References}

1 The polycystic kidney disease 1 gene encodes a $14 \mathrm{~kb}$ transcript and lies within a duplicated region on chromosome 16. The European Polycystic Kidney Disease Consortium. Cell 1994;77:881-94.

2 Mochizuki T, Wu G, Hayashi T, Xenophontos SL et al. PKD2, a gene for polycystic kidney disease that encodes an integral membrane protein. Science 1996; 272:1339-42.

3 Igarashi P, Somlo S. Genetics and pathogenesis of polycystic kidney disease. Review. J Am Soc Nephrol 2002;13:2384-98.

4 Hateboer N, van Dijk M, Bogdanova N, Coto E et al. Comparison of phenotypes of polycystic kidney disease types 1 and 2 . European PKD1-PKD2 Study Group. Lancet 1999;353:103-7.

5 Ravine D, Gibson RN, Walker RG, Sheffield LJ et al. Evaluation of ultrasonographic diagnostic criteria for autosomal dominant polycystic kidney disease 1. Lancet 1994; 343:824-7.

6 Klahr S, Breyer JA, Beck GJ, Dennis VW et al. Dietary protein restriction, blood pressure control, and the progression of polycystic kidney disease. Modification of Diet in Renal Disease Study Group. J Am Soc Nephrol 1995;5:2037-47.

7 Fick GM, Johnson AM, Hammond WS, Gabow PA. Causes of death in autosomal dominant polycystic kidney disease. Review. J Am Soc Nephrol 1995;5:2048-56.

8 Schrier R, McFann K, Johnson A, Chapman A et al. Cardiac and renal effects of standard versus rigorous blood pressure control in autosomal-dominant polycystic kidney disease: results of a seven-year prospective randomized study. J Am Soc Nephrol 2002;13: 1733-9.

9 Taylor JE, Calne RY, Stewart WK. Massive cystic hepatomegaly in a female patient with polycystic disease treated by combined hepatic and renal transplantation. QJM 1991;80:771-5.

10 Pirson Y, Chauveau D, Torres V. Management of cerebral aneurysms in autosomal dominant polycystic kidney disease. Review. J Am Soc Nephrol 2002;13:269-76.

11 Sharp CK, Zeligman BE, Johnson AM, Duley I, Gabow PA. Evaluation of colonic diverticular disease in autosomal dominant polycystic kidney disease without end-stage renal disease. Am J Kidney Dis 1999;34: $863-8$.

12 Stewart JH, Buccianti G, Agodoa L, Gellert $\mathrm{R}$ et al. Cancers of the kidney and urinary tract in patients on dialysis for end-stage renal disease: analysis of data from the United States, Europe, and Australia and New Zealand. J Am Soc Nephrol 2003;14: 197-207.

13 Keith DS, Torres VE, King BF, Zincki H, Farrow GM. Renal cell carcinoma in autosomal dominant polycystic kidney disease. Review. J Am Soc Nephrol 1994;4:1661-9.

14 Davis ID, MacRae Dell K, Sweeney WE, Avner ED. Can progression of autosomal dominant or autosomal recessive polycystic kidney disease be prevented? Review. Semin Nephrol 2001;21:430-40.

15 Schrier RW, McFann KK, Johnson AM. Epidemiological study of kidney survival in autosomal dominant polycystic kidney disease. Kidney Int 2003;63:678-85.

16 Yoder BK, Hou X, Guay-Woodford LM. The polycystic kidney disease proteins, polycystin-1, polycystin-2, polaris and cystin, are co-localized in renal cilia. J Am Soc Nephrol 2002;13:2508-16. 